\title{
The utility of repeated computed tomography to track a foreign body penetrating the esophagus to the level of the thyroid gland
}

\author{
Hai-hong Chen • Ling-Xiang Ruan • \\ Shui-Hong Zhou $\cdot$ Shen-Qing Wang
}

Received: 17 December 2012/ Accepted: 25 September 2013/Published online: 24 October 2013

(C) The Author(s) 2013. This article is published with open access at Springerlink.com

\begin{abstract}
Objective Foreign body (FB) ingestion is a common problem in otolaryngology. One uncommon complication of FB ingestion is penetration to the level of the thyroid gland. To our knowledge, only 21 such cases have been reported in the literature. Here, we report a case of an esophageal FB penetrating to the level of the right thyroid gland.

Case report The patient was a 38-year-old woman in whom an esophageal FB penetrated to the level of the right thyroid gland. We traced the path to the thyroid gland using repeated computed tomography (CT) scans and demonstrated the importance of multiplanar reconstruction in locating the FB and formulating a precise surgical plan. Conclusions To our knowledge, this is the first report of repeat $\mathrm{CT}$ scans being used to demonstrate the migratory route, over time, of a FB penetrating through the esophagus to the level of the thyroid gland. Our results suggest that multiplanar reconstruction may play a key role in the precise diagnosis of a FB at the level of the thyroid gland and may help surgeons choose the best approach for removal.
\end{abstract}

Keywords Foreign body - Migration · Thyroid gland $\cdot$ Computed tomography $\cdot$ Surgery

H. Chen · S.-H. Zhou $(\varangle) \cdot$ S.-Q. Wang

Department of Otolaryngology, The First Affiliated Hospital, College of Medicine, Zhejiang University, Hangzhou 310003, China

e-mail: zhouyunzhoush@163.com

L.-X. Ruan

Department of Radiology, The First Affiliated Hospital, College of Medicine, Zhejiang University, Hangzhou 310003, China

\section{Introduction}

Foreign body (FB) ingestion is a common problem in otolaryngology practices [1]. One uncommon complication of FB ingestion is penetration to the level of the thyroid gland and, to our knowledge only 21 such cases have been reported [1-17]. If left untreated, serious complications associated with high risks of morbidity and mortality can develop, such as periesophagitis, periesophageal abscess, mediastinitis, aortoesophageal fistula, innominate esophageal fistula, and carotid rupture [3, 4]. Therefore, prompt diagnosis is essential in the management of perforating FBs [3]. Most FBs can be located using computed tomography (CT). The CT results can then be used to guide the approach toward FB removal, although the path by which esophageal FBs migrate to the level of the thyroid gland remains unclear.

Here, we report a patient in whom an esophageal FB penetrated to the level of the right thyroid gland. We traced the path of the FB via repeated CT scans. Our case demonstrates the importance of multiplanar reconstruction (MPR) in locating an FB and formulating a precise surgical plan.

\section{Case report}

A 38-year-old woman presented to the emergency department with a history of sudden painful dysphagia after accidentally ingesting a FB during dinner $2 \mathrm{~h}$ earlier. Indirect laryngoscopy showed no FB in the oral cavity, pharynx, or larynx. A CT scan $1 \mathrm{~h}$ later revealed a hyperdense FB obliquely traversing the right lateral wall of the esophagus, and MPR clearly showed an approximately 2.8-cm-long oblique radiopaque linear FB in the cervical 
A

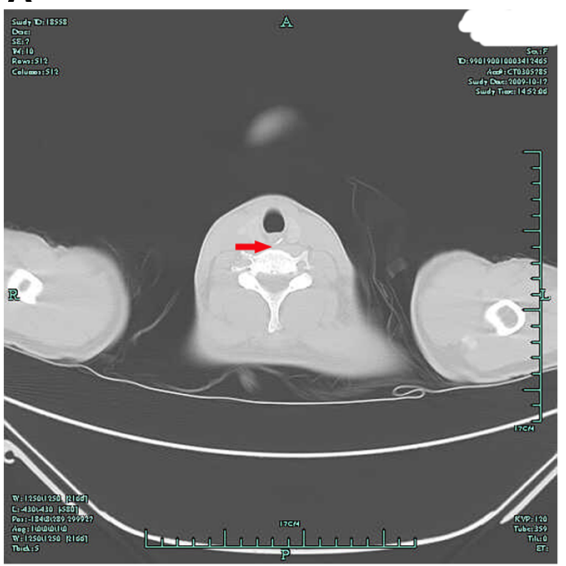

D

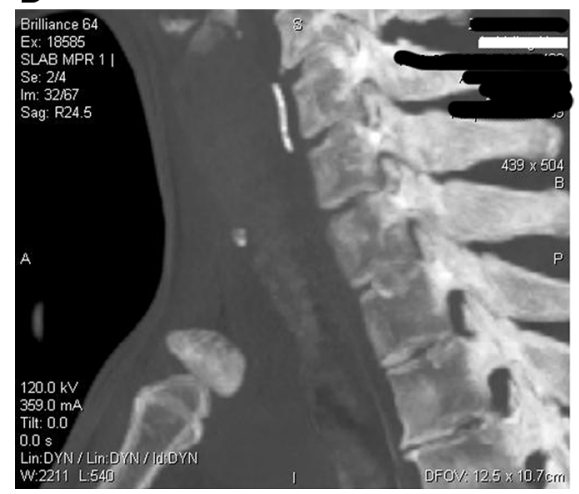

B

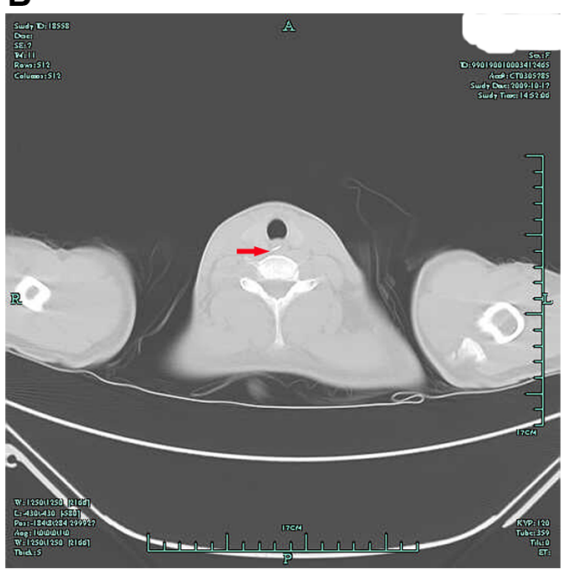

E

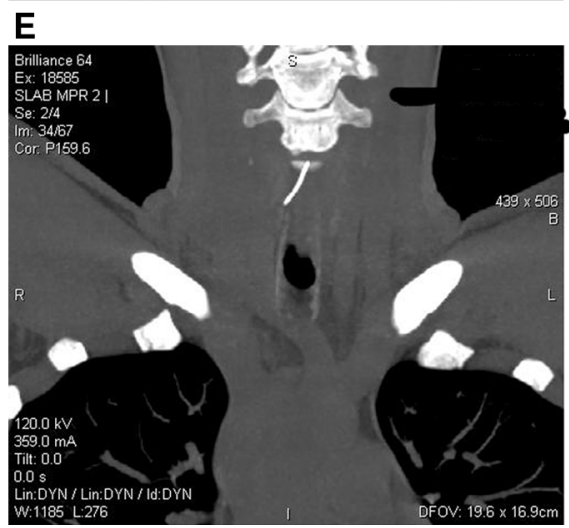

C

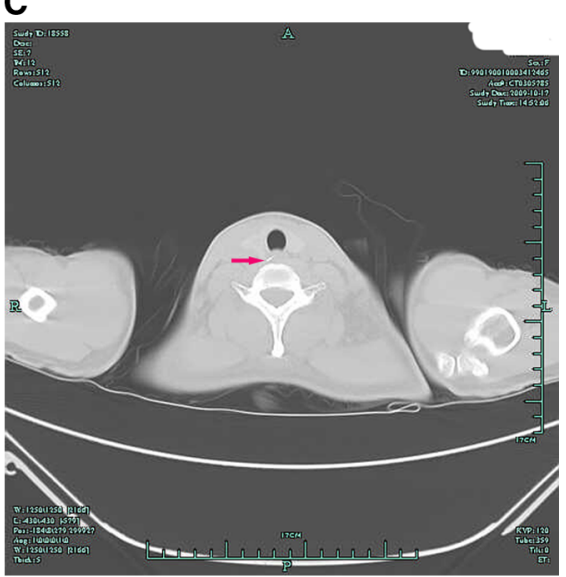

Fig. 1 A 38-year-old woman with cervical pain and difficulty swallowing after accidentally ingesting a foreign body (FB) $2 \mathrm{~h}$ previously. a-c Plain transverse images showing the FB in the cervical esophagus (arrow), d sagittal multiplanar reconstruction (MPR) clearly revealing an oblique radio-opaque linear FB approximately $2.8 \mathrm{~cm}$ long located in the cervical esophagus in front of the sixth cervical vertebra (C6), with the long axes of the FB and esophagus making an angle of approximately $13^{\circ}$, e coronal MPR revealing that the FB had traversed the right wall of the esophagus esophagus, in front of the sixth cervical vertebra (C6). The long axes of the FB and esophagus formed an angle of approximately $13^{\circ}$ (Fig. 1). Rigid esophagoscopy was performed under general anesthesia $9 \mathrm{~h}$ later, but the FB was not found despite a careful search. The day after ingestion, the patient's odynophagia was relieved. However, a plain radiograph revealed a linear hyperdense mass in front of the C7 and T1 bodies (Fig. 2). A subsequent CT scan of the neck the next day showed that the FB had penetrated the esophageal wall (Fig. 3a, b, c). MPR demonstrated that the FB had moved into the adipose space in the right paraesophageal area and downward in front of $\mathrm{C} 7$. Its long axis now paralleled that of the esophagus (Fig. 3d, e). It was impossible to remove the FB via esophagoscopy, so we suggested removing it using an external approach via lateral neck exploration. However, the patient refused surgery. The patient was given antibiotics for several days, and her symptoms improved greatly; she had no difficulty swallowing. Ten days after ingestion, a CT scan indicated that the FB had migrated to near the right thyroid gland (Fig. 4). The patient underwent an open right neck exploration. Based on the $\mathrm{CT}$ findings, the right thyroid gland was exposed and carefully pulled upward; the FB, a fine wire measuring $2.8 \mathrm{~cm}$ in length, was located just behind it (Fig. 5). The patient had an uneventful postoperative period.

\section{Discussion}

A migratory esophageal FB is uncommon, and migration of these FBs to the level of thyroid gland is rare. A search of the Medline database using the method set out by Hohman et al. [5] revealed 22 cases of FBs that had migrated to the level of the thyroid gland, including our case (Table 1). Interestingly, all of the patients were adult women, with a mean age of 50.3 (range 21-73) years. The time from ingestion to presentation varied from $2 \mathrm{~h}$ to 1 year, with a 
A

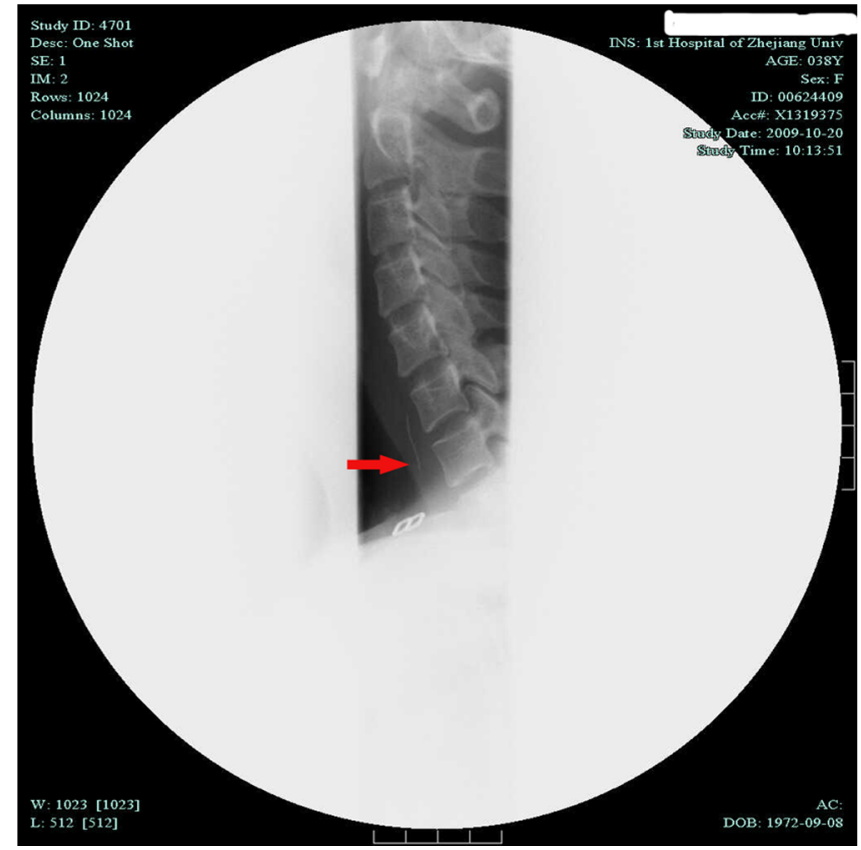

B

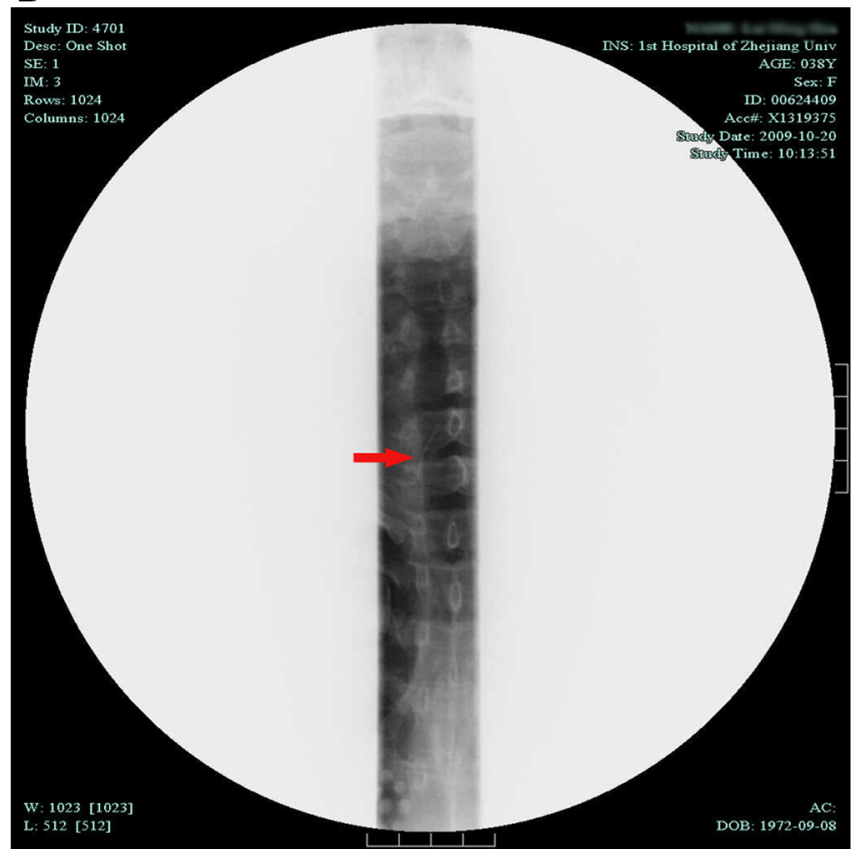

Fig. 2 A plain radiograph showing a linear hyperdense area (arrow) in front of the C7 and T1 bodies: a sagittal image, b coronal image

mean delay in presentation of 20 days; this was longer than the period reported by Hohman et al. [5]. Taken together, these FBs consisted of 16 fish bones, two chicken bones, and four metal wires or bristles; of these, 12 and ten were located at the level of the right thyroid gland and left thyroid gland, respectively, showing an almost equal distribution.

To our knowledge, this is the first report of CT being used to demonstrate the migratory path, over time, of a FB penetrating the esophagus and traveling to the level of the thyroid gland. The FB began to migrate in the esophagus within $10 \mathrm{~h}$ and penetrated the esophageal wall into the surrounding adipose space within about 1 day. The contraction of the cricopharyngeal muscle during swallowing probably played a significant role in this process [11]. Given the barrier roles of the anterior (trachea) and posterior (cervical vertebra) regions of the esophageal wall [11], a FB may penetrate only through the lateral wall of the esophagus toward the level of the right or left thyroid gland (no predilection). If untreated, the FB will continue to migrate after swallowing. In our case, the FB took 10 days to travel to the level of the right thyroid gland. The nature of the FB and the angle between the FB and esophageal wall may also play a role in the migration process. All of the cases reported to date involved hard, pointed FBs [1-17], with the FBs having a mean length of $2.8(2.0-4.1) \mathrm{cm}$. Our patient swallowed a fine, pointed wire. The nature of the FB may facilitate transmural esophageal penetration and subsequent migration to the level of the thyroid gland [11]. Al-Sebeih et al. [17] suggested that the more horizontally oriented and sharper the $\mathrm{FB}$ is, the higher the risk of penetration. However, we found that the FB migrated easily with a small acute angle between the long axes of the FB and esophagus. Of course, we cannot rule out the possibility that pushing on the rigid esophagoscope facilitated the migration. If a FB is not removed, it will continue to migrate and may cause serious complications, such as aortoesophageal fistula or carotid rupture $[3,8]$. Furthermore, a FB can introduce bacteria into the soft tissues of the neck, causing suppurative complications such as parapharyngeal or retropharyngeal abscesses and mediastinitis [3, 8]. Fortunately, no serious complications occurred in our case.

For the above-mentioned reasons, it is very important to remove a FB in a timely manner. However, it is difficult to locate a migrated short fine wire in the neck via an open approach. The main difficulty is localizing the FB in the soft tissues, after which removal is usually simple. Traditional approaches to locate an impacted FB generally include using plain films of the neck and chest, barium swallow, and direct esophagoscopic examination. A plain radiograph may also indicate the level of the FB and may be useful in a follow-up context, as in the case of our patient. However, plain radiography usually affords only 

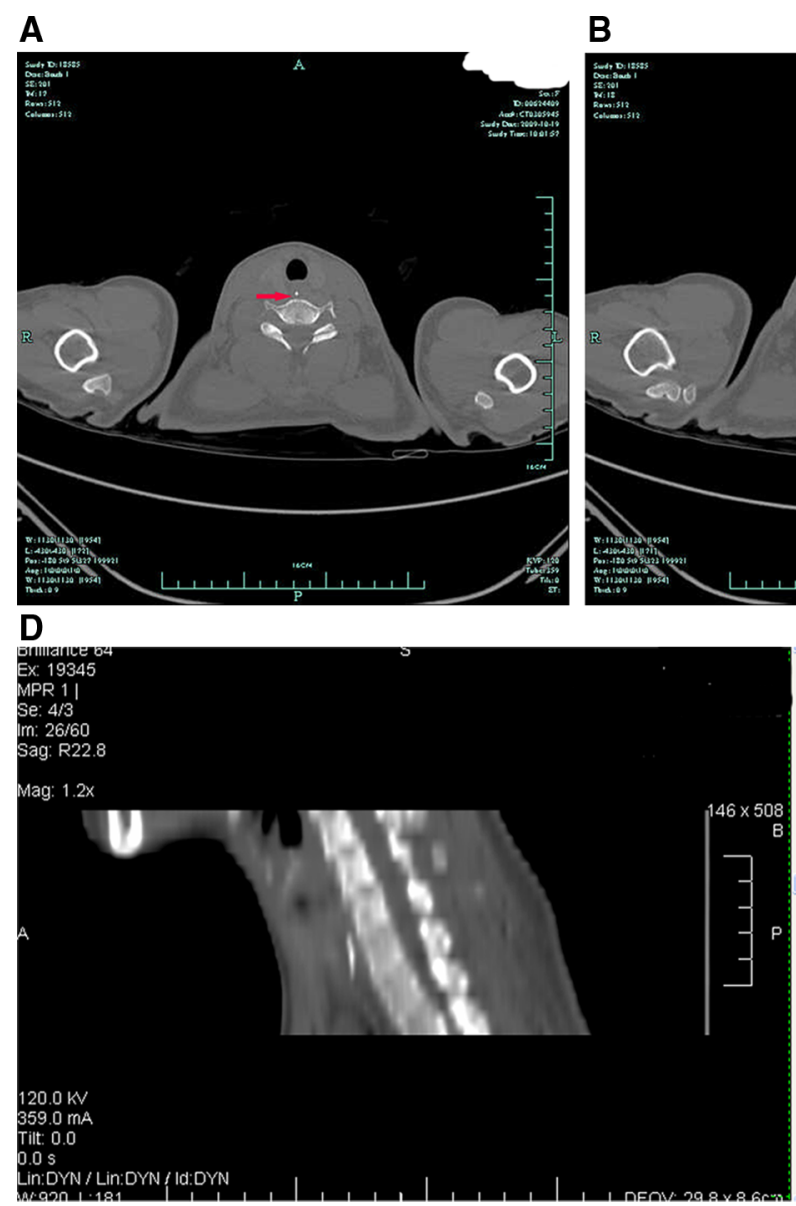

Fig. 3 The day after ingestion. A computed tomography (CT) scan revealed a linear hyperdensity located next to the esophageal lumen, indicating that the FB had penetrated the esophageal wall. a-c Plain transverse images revealing the FB (arrow) in the cervical esophagus, d sagittal MPR revealing that the FB had moved downward and was
B

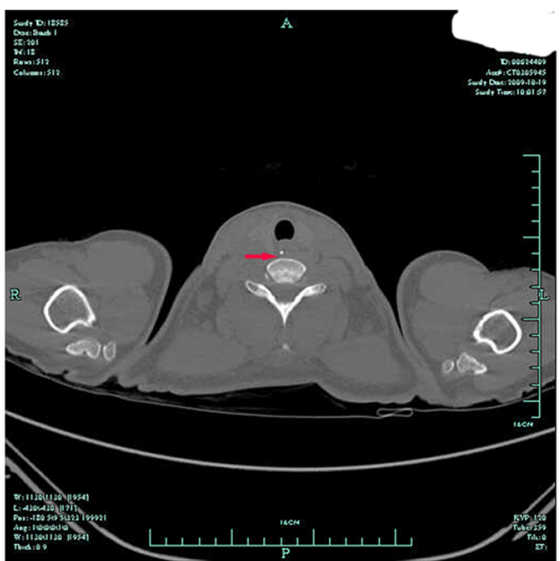

$\mathbf{E}$
C
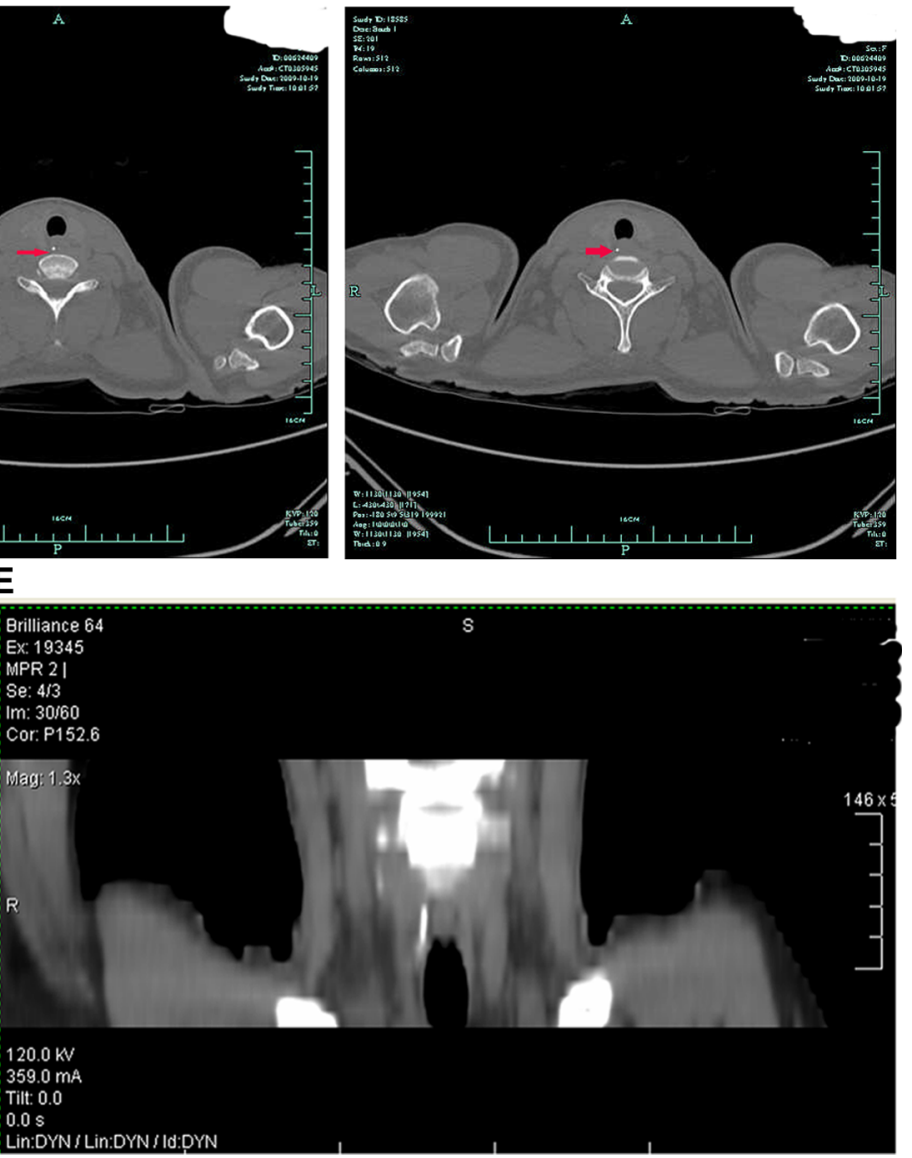

now in front of $\mathrm{C} 7$, with its long axis paralleling the longitudinal esophageal axis, e coronal MPR revealing that the FB had moved into the right paraesophageal adipose space and that its long axis now paralleled the esophageal longitudinal axis

poor sensitivity when the $\mathrm{FB}$ is a fish or chicken bone because bones are rarely visible on radiographs $[18,19]$. A plain radiograph is therefore suitable only for searching for the more radiodense FBs.

Barium swallows have yielded different results in different cases [20,21]. Many endoscopists emphasize their negative features. Mosca et al. [21] reported that barium involves a risk of aspiration, can delay an emergency endoscopic procedure, and can complicate removal of a FB by interfering with endoscopic visualization. Therefore, in some hospitals, the patient is sent immediately for esophagoscopy, without a diagnostic imaging assessment [22]. Esophagoscopy, even those using flexible endoscopes, is an invasive technique with a potential morbidity risk [22]. CT is an important diagnostic technique in cases of migrating esophageal FBs. Although a plain radiograph may show the level of the FB, we found that repeated CT scans showed the migratory path, over time, of the FB more clearly and that MPR (commonly employed in the evaluation of various diseases, such as fractures of the skull base [23]) may be helpful when used to diagnose a FB at the level of the thyroid gland. Using axial and coronal reconstruction, MPR can show the shape, size, length, location, and direction of the FB, thereby providing important information to guide the surgical approach. Using various imaging angles in multiple series of images can reveal the anatomical relationship between an impacted FB and vital structures of the head and neck, such as the trachea, cervical vertebrae, thyroid gland, and arteries of the neck. These data can help surgeons choose the best approach to remove the FB and reduce surgical complications. We exposed the right thyroid gland directly through a lateral neck incision and removed the fine wire successfully.

In conclusion, to our knowledge, this is the first demonstration of the migratory trace and time of a FB penetrating through the esophagus to the level of the thyroid gland using 

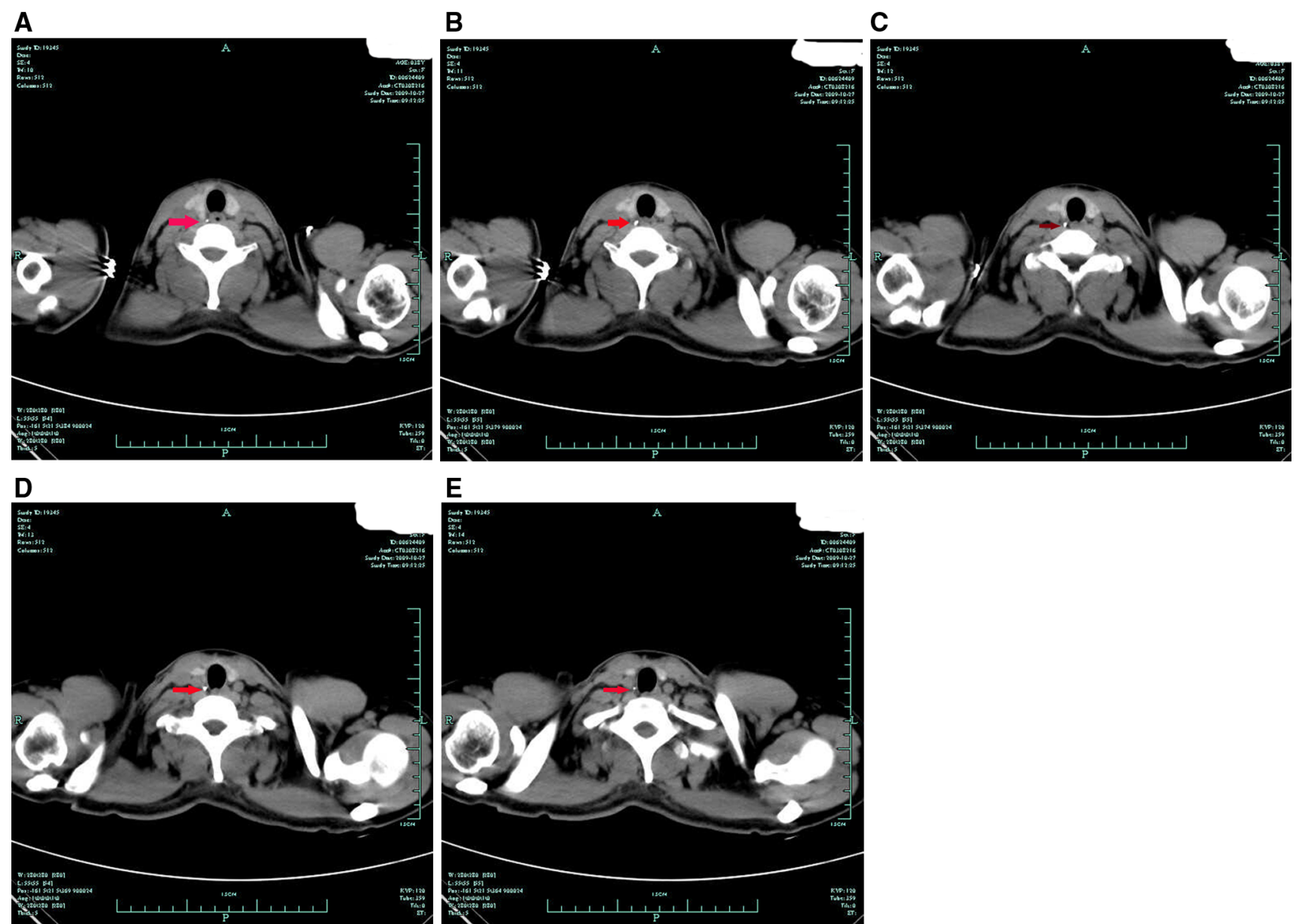

Fig. 4 Transverse imaging at 10 days after ingestion revealing a linear hyperdense area (arrow) located behind and at the level of the right thyroid gland $(\mathbf{a}-\mathbf{e})$

Fig. 5 Intraoperative photograph of the FB extraction (a). A fine wire (arrows), measuring $2.8 \mathrm{~cm}$ in length, was identified behind the right thyroid gland (b)
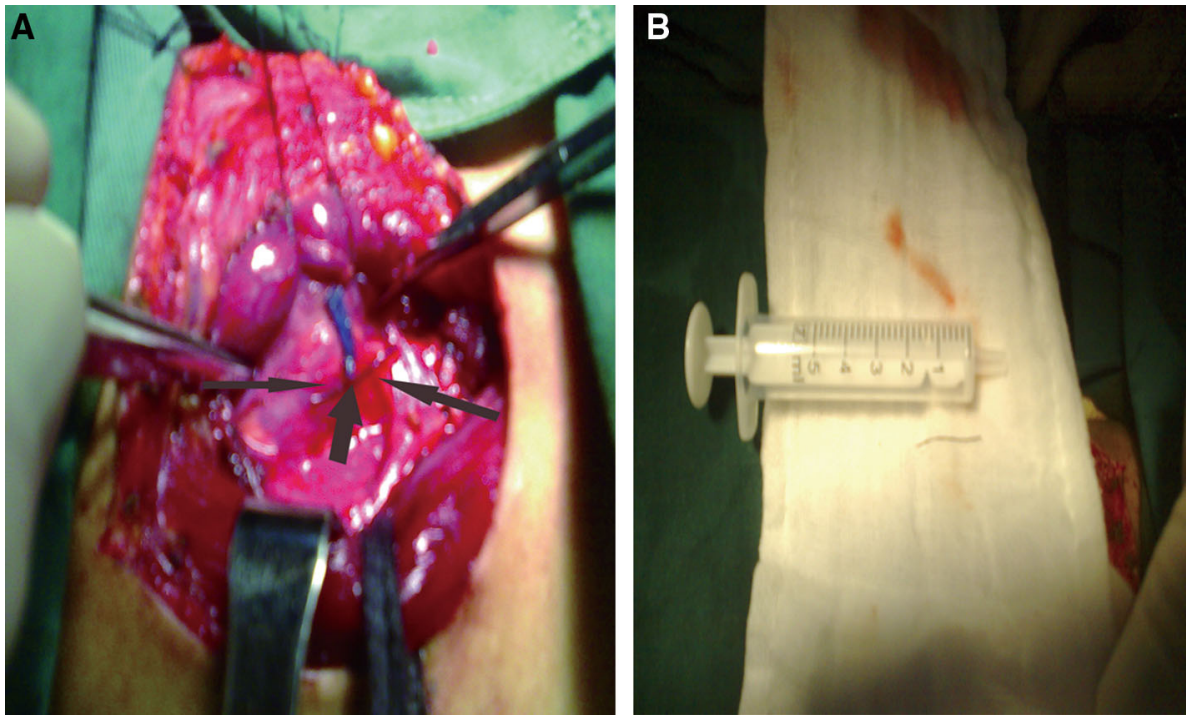


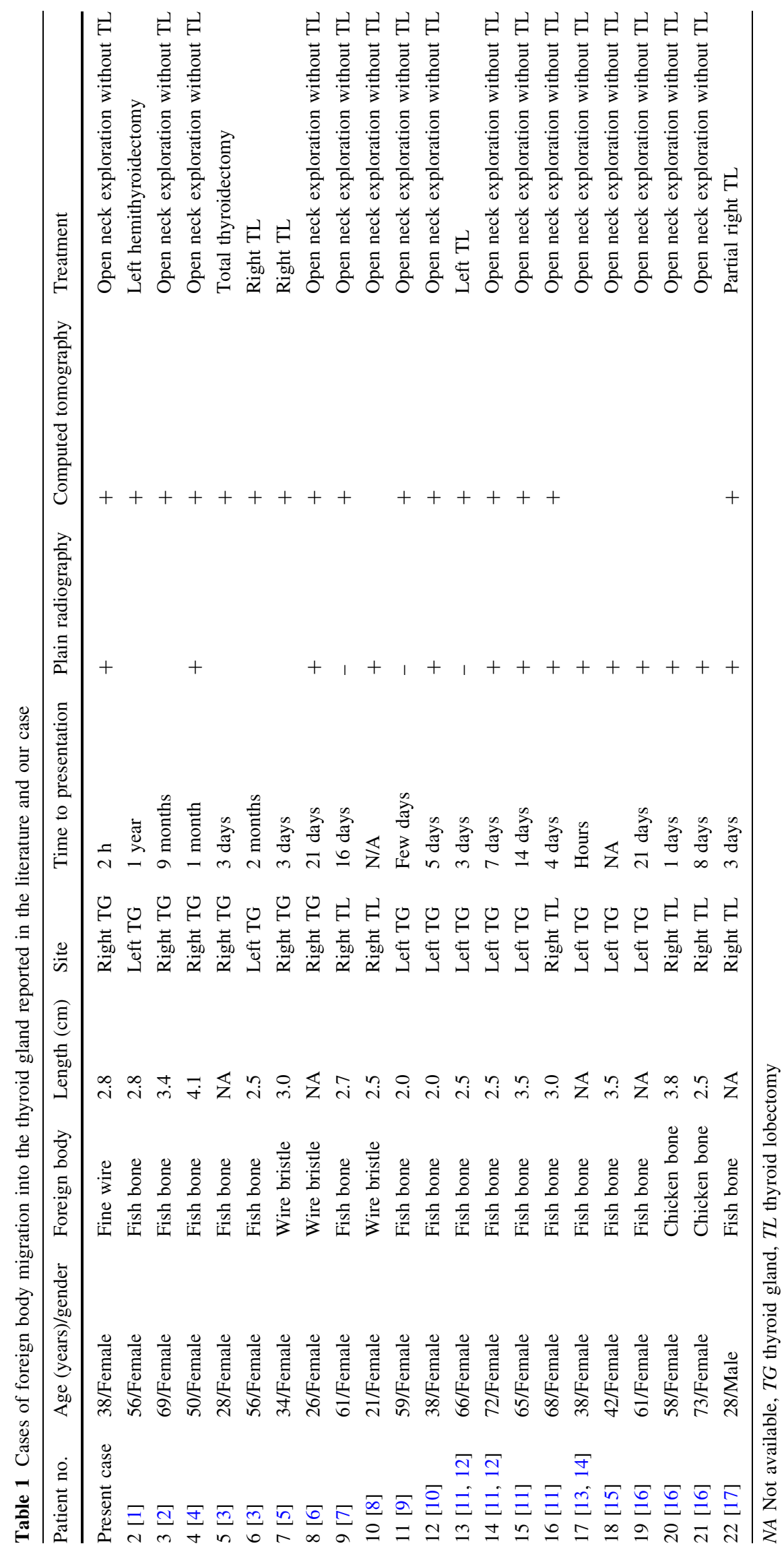


repeated CT. We also demonstrated that MPR can play a key role in the diagnosis of a FB at the level of the thyroid gland and help surgeons choose the best approach to remove the FB and reduce surgical complications.

\section{Conflict of interest None.}

Open Access This article is distributed under the terms of the Creative Commons Attribution License which permits any use, distribution, and reproduction in any medium, provided the original author(s) and the source are credited.

\section{References}

1. Watanabe K, Amano M, Nakanome A, Saito D, Hashimoto S. The prolonged presence of a fish bone in the neck. Tohoku J Exp Med. 2012;227:49-52.

2. Ohbuchi T, Tabata T, Nguyen KH, Ohkubo J, Katoh A, Suzuki H. Thyroid gland cutaneous fistula secondary to a migratory fish bone: a case report. J Med Case Rep. 2012;6:140.

3. Al-Sebeih K, Abu-Shara KA, Sobeih A. Extra luminal perforation complicating foreign bodies in the upper aero digestive tract. Ann Otol Rhinol Laryngol. 2010;119:284-8.

4. Chen CY, Peng JP. Esophageal fish bone migration induced thyroid abscess: case report and review of the literature. Am J Otolaryngol. 2011;32:253-5.

5. Hohman MH, Harsha WJ, Peterson KL. Migration of ingested foreign bodies into the thyroid gland: literature review and case report. Ann Otol Rhinol Laryngol. 2010;119:93-8.

6. Zohra T, Ikram M, Iqbal M, Akhtar S, Abbas SA. Migrating foreign body in the thyroid gland, an unusual case. J Ayub Med Coll Abbottabad. 2006;18:65-6.

7. Masuda M, Honda T, Hayashida M, Samejima Y, Yumoto E. A case of migratory fish bone in the thyroid gland. Auris Nasus Larynx. 2006;33:113-6.

8. Astl J, Belsan T, Veselý D. Penetrating esophageal foreign body: metallic wire in the thyroid gland. $\mathrm{J}$ Otolaryngol. 2005;34:209-11.

9. Sergi B, Contucci A, Celidonio BA, De Gaetano AM, Lemmo GF. Fish bone migration through the thyroid gland in the neck. J Otolaryngol. 2002;31:396-8.
10. Arumainathan UD, Lwin S, Suan TL, Raman R. Removal of a fish bone in the thyroid gland without the need for thyroid lobectomy. Ear Nose Throat J. 2000;79:314-5.

11. Goh YH, Tan NG. Penetrating oesophageal foreign bodies in the thyroid gland. J Laryngol Otol. 1999;113:769-71.

12. Foo TH. Migratory fish bone in the thyroid gland. Singapore Med J. 1993;34:142-4.

13. Coret A, Heyman Z, Bendet E, Amitai M, Itzchak I, Kronberg J. Thyroid abscess resulting from transesophageal migration of a fish bone: ultrasound appearance. J Clin Ultrasound. $1993 ; 21: 152-4$.

14. Bendet E, Horowitz Z, Heyman Z, Faibel M, Kronenberg J. Migration of fishbone following penetration of the cervical esophagus presenting as a thyroid mass. Auris Nasus Larynx. 1992;19:193-7.

15. Al Muhanna A, Abu Chra KA, Dashti H, Behbehani A, Al-Naqeeb N. Thyroid lobectomy for removal of a fish bone. J Laryngol Otol. 1990;104:511-2.

16. Jemerin EE, Aronoff JS. Foreign body in thyroid following perforation of esophagus. Surgery. 1949;25:52-9.

17. Al-Sebeih K, Valvoda M, Sobeih A, Al-Sihan M. Perforating and migrating pharyngoesophageal foreign bodies: a series of 5 patients. Ear Nose Throat J. 2006;85:600-3.

18. Lue AJ, Fang WD, Manolidis S. Use of plain radiography and computed tomography to identify fish bone foreign bodies. Otolaryngol Head Neck Surg. 2000;123:435-8.

19. Evans RM, Ahuja A, Rhys Williams S, Van Hasselt CA. The lateral neck radiograph in suspected impacted fish bones-does it have a role? Clin Radiol. 1992;46:121-3.

20. Akazawa Y, Watanabe S, Nobukiyo S, Iwatake H, Seki Y, Umehara $\mathrm{T}$, et al. The management of possible fishbone ingestion. Auris Nasus Larynx. 2004;31:413-6.

21. Mosca S, Manes G, Martino L, Amitrano L, Bottino V, Bove A, et al. Endoscopic management of foreign bodies in the upper gastrointestinal tract: report on a series of 414 adult patients. Endoscopy. 2001;33:692-6.

22. De Lucas Marco E, Sádaba P, Lastra García-Barón P, RuizDelgado ML, González Sánchez F, Ortiz A, et al. Value of helical computed tomography in the management of upper esophageal foreign bodies. Acta Radiol. 2004;45:369-74.

23. Wang $\mathrm{H}, \mathrm{Xu} \mathrm{M}$, Liang $\mathrm{H}, \mathrm{Xu} \mathrm{L}$. Comparison of CT and MRI in diagnosis of cerebrospinal leak induced by multiple fractures of skull base. Radiol Oncol. 2011;45:91-6. 